\title{
SISTEMAS AQUOSOS BIFÁSICOS: FUNDAMENTOS E APLICAÇÕES PARA PARTIÇÃO/PURIFICAÇÃO DE PROTEÍNAS
}

Luis Henrique Mendes da Silva*

Departamento de Química, Centro de Ciências Exatas e Tecnológicas, Universidade Federal de Viçosa, 36571-000 Viçosa - MG, Brasil Watson Loh

Instituto de Química, Universidade Estadual de Campinas, CP 6154, 13084-971 Campinas - SP, Brasil

Recebido em 14/4/05; aceito em 16/12/05; publicado na web em 14/6/06

\begin{abstract}
AQUEOUS TWO-PHASE SYSTEMS: FUNDAMENTALS AND APPLICATIONS FOR PARTITIONING/PURIFICATION OF PROTEINS. By the year 2005 the world biochemical market will reach an estimated $\$ 100$ billion and separation processes are a vital link between lab discoveries and the fulfillment of this commercialization potential. The practical application of aqueous twophase systems (ATPS) to extraction processes has been exploited for several years for the recovery of biological products. Unfortunately, this has not resulted in an extensive presence of the technique in commercial processes. In this paper a critical overview of the fundamental thermodynamic properties related to formation of aqueous two-phase systems and their application to extraction and purification of bioparticules is presented.
\end{abstract}

Keywords: aqueous two phase system; proteins; biotechnology.

\section{INTRODUÇÃO}

Sistemas contendo duas ou mais fases líquidas em equilíbrio termodinâmico são muito úteis para aplicações em extração e/ou purificação de compostos presentes em inúmeros processos tecno$\operatorname{lógicos}^{1,2}$. Porém, para aplicação desta técnica de extração líquidolíquido para a obtenção de compostos com importância biotecnológica (proteínas, enzimas, células, vírus, organelas, etc.), faz-se necessária a utilização de sistemas em que as duas fases guardem semelhanças, em termos das propriedades físico-químicas, com o meio aquoso presente nos seres vivos ${ }^{3}$. Assim, a aplicação dos clássicos sistemas bifásicos solvente orgânico/água para extração de uma proteína seria inviável, na maioria dos casos pois, com uma eventual concentração da proteína na fase orgânica, ocorreria um processo de desnaturação, com a conseqüente perda de atividade da biomolécula.

Neste sentido, os sistemas denominados aquosos bifásicos (SABs ou, em inglês, ATPS) desempenham um papel estratégico pois, como veremos em maiores detalhes a seguir, as duas fases são formadas predominantemente (60-95\%) por água.

A formação dos SABs é conhecida desde o final do século 19. Em 1896, Beijerinck ${ }^{4,5}$ descobriu que soluções aquosas de gelatina e agar ou gelatina e amido solúvel, misturadas em uma dada faixa de temperatura e concentração, formavam misturas turvas que, em repouso, separavam espontaneamente em duas fases líquidas límpidas. A fase mais densa era enriquecida em agar (ou amido) e a fase superior em gelatina, sendo que a água era o componente majoritário em ambas as fases.

Posteriormente, Ostwald e Hertel ${ }^{6,7}$ continuaram os estudos sobre esses sistemas e verificaram que amidos provenientes de origens distintas (arroz, milho, etc.), isto é, possuindo diferentes frações de amilose e amilopectina, produziam diferentes diagramas de fase. Esses resultados mostravam a grande influência que pequenas variações nas interações intermoleculares têm sobre as composições das fases em equilíbrio.

Com objetivo de verificar uma possível generalidade do fenômeno de separação de fase em sistemas contendo macromoléculas, Dobry

*e-mail: luhen@ufv.br e Boyer-Kawenoki ${ }^{8,9}$, no final da década de 40, estudaram a miscibilidade de um grande número de diferentes pares de polímeros, dissolvidos em solventes orgânicos ou em soluções aquosas. Estes autores constataram que dos 35 pares de macromoléculas estudados, apenas 4 não produziram a formação das duas fases, e puderam concluir que a incompatibilidade entre polímeros era um fenômeno geral.

Porém, foi apenas com os trabalhos de Per-Åke Albertsson ${ }^{10}$, em meados da década de 50, que ficou evidente para a comunidade científica a grande potencialidade de aplicação destes sistemas à partição/purificação de materiais biológicos, desde proteínas até células. Como o solvente é a água em ambas as fases e geralmente se encontra presente em grande quantidade, estes sistemas fornecem um ambiente ameno para as biomoléculas evitando, assim, mudanças em suas estruturas e perda das atividades biológicas.

Outra propriedade termodinâmica importante associada aos SABs, e que lhes dá uma vantagem significativa quando comparados aos clássicos sistemas água-óleo, é possuírem regiões interfaciais com baixo valor de tensão interfacial, $\gamma^{11-13}$. Para aplicação em processos de separação de biomoléculas, este pequeno valor de excesso de energia livre de Gibbs por unidade de área possibilita a transferência das biopartículas pela interface com um risco mínimo de ocorrerem alterações estruturais nas proteínas, células e membranas particionadas ${ }^{14}$.

A natureza química dos componentes formadores das fases tem forte impacto nas propriedades interfaciais dos sistemas aquosos bifásicos. Além de influenciar no coeficiente de partição dos solutos, a tensão interfacial contribui para outras propriedades do sistema, tais como a estabilidade cinética e termodinâmica da dispersão, cinética de partição, etc ${ }^{15}$.

A primeira publicação relatando resultados de tensão interfacial de SABs foi de Ryden e Albertsson, em $1971^{16}$. O sistema bifásico estudado era formado por PEG e Dextrana. Neste trabalho já era relatado um comportamento característico de $\gamma$ em função de duas variáveis termodinâmicas; o comprimento da linha de amarração (CLA) e a massa molar dos dois polímeros. Estes autores mostraram existir uma relação simples entre a tensão interfacial e estas duas características dos SABs formados, conforme Equação 1 
$\gamma=\mathrm{a}(\mathrm{CLA})^{\mathrm{b}}$

onde a e b são constantes dependentes da massa molar das macromoléculas formadoras dos SABs. Os valores de $\gamma$ permaneceram entre $6.55 \times 10^{-2}$ e $4.6 \times 10^{-4} \mathrm{mN} \mathrm{m}^{-1}$, sendo que os valores menores pertenciam a composições mais próximas ao ponto crítico. Em 1984, Bamberg et al. ${ }^{17}$ estudaram o mesmo sistema e propuseram uma relação exponencial entre a tensão interfacial e a diferença de composição entre as duas fases, expressa pelo comprimento da linha de amarração (CLA)

$\gamma=\operatorname{ce}^{\mathrm{d}(\mathrm{CLA})}$

em que c e d são constantes. Uma limitação clara da Equação 2 é o fato de que ela prevê um valor finito para a tensão interfacial quando o sistema se encontra na composição do ponto crítico e isto naturalmente não faz sentido. No ponto crítico, acabam as diferenças entre todas as propriedades termodinâmicas intensivas de cada fase e, conseqüientemente, o valor da tensão interfacial se iguala a zero.

A partir destes trabalhos pioneiros, centenas de outros foram realizados, enfocando, em sua quase totalidade, a descoberta, caracterização e aplicação de novos sistemas à separação de solutos específi$\cos ^{1,10,18,19}$. A Tabela 1 exemplifica alguns sistemas descobertos e aplicados com sucesso na purificação de algumas biopartículas.

Atualmente, os sistemas aquosos bifásicos são aplicados mais eficientemente em etapas iniciais dos processos de purificação, mas em casos raros podem até mesmo substituir os clássicos sistemas cromatográficos.

Tabela 1. Sistemas aquosos bifásicos formados por diferentes componentes

\begin{tabular}{ll}
\hline Componente 1 & Componente 2 \\
\hline Poli( óxido de etileno) & Poli(óxido de propileno) \\
Poli(óxido de etileno) & Dextrana \\
Poli (óxido de etileno) & Poli(N-vinil-2-pirrolidona) \\
Poli(óxido de etileno) & $\mathrm{Na}_{2} \mathrm{SO}_{4}$ \\
Copolímero Poli(óxido de etileno)- & $\mathrm{Maltodextrina}$ \\
poli(óxido de propileno)- & \\
poli(óxido de etileno) & \\
$\begin{array}{l}\text { Dextrana sulfonato de sódio } \\
\text { Poli(óxido de etileno) }\end{array}$ & Etil-hidroxietilcelulose- $\mathrm{NaCl}$ \\
Dextrana sulfonato de sódio & $\mathrm{Citrato} \mathrm{de} \mathrm{sódio}$ \\
\hline
\end{tabular}

\section{FUNDAMENTOS SOBRE A FORMAÇÃO DOS SABs}

A formação de duas fases durante o processo de mistura de soluções aquosas de dois polímeros (ou de um polímero e de um sal) dependerá das interações intermoleculares - expressas em termos da energia livre - entre os constituintes formadores do sistema. Serão também estas mesmas interações as responsáveis por todas as propriedades físico-químicas presentes nas duas fases, como por ex., a distribuição dos diferentes componentes no sistema, da relação de volumes entre as fases, da diferença de potencial elétrico e do excesso de energia livre associados à interface, do coeficiente de partição de um soluto específico, etc.

Para cada sistema, teremos pares de componentes que possuirão interações termodinamicamente favoráveis e pares que gerarão interações que aumentarão a energia livre do sistema e, por isto, serão consideradas desfavoráveis. Naturalmente estas interações serão dependentes do estado termodinâmico do sistema, isto é, da composição, temperatura e pressão.
Em um dos sistemas aquosos bifásicos mais estudados e aplicados, o SABs formado por dextrana-PEO- $\mathrm{H}_{2} \mathrm{O}$, geralmente se atribui a separação do sistema em duas fases ao surgimento de interações desfavoráveis quando segmentos de dextrana entram em contato com segmentos do poli(óxido de etileno) ${ }^{20}$. Loh e da Silva ${ }^{21}$, utilizando medidas calorimétricas, mostraram que esta interação repulsiva entre PEO e dextrana possui contribuição entálpica muito pequena, sendo predominantemente de natureza entrópica, isto é, oriunda de efeito de volume de exclusão ${ }^{22}$, entropia configuracional ${ }^{23}$, etc.

Em outros casos pode ser exatamente o contrário. Existem SABs que se formam porque existe uma forte interação entre segmentos de diferentes polímeros. Neste caso, a diminuição da energia livre pode ser devido a interações eletrostáticas entre cargas positivas presentes em um dado polímero com cargas negativas distribuídas ao longo da cadeia de uma outra macromolécula ${ }^{24}$, ou à formação de ligações de hidrogênio entre os segmentos, gerando complexos de coacervação ${ }^{25}$. É interessante destacar que à medida que o número de diferentes componentes aumenta, abrem-se possibilidades de se formarem sistemas polifásicos. Em 1998, foi descoberto que a adição de $\mathrm{CH}_{2} \mathrm{Cl}_{2}$ a um sistema aquoso bifásico formado por $\mathrm{Na}_{2} \mathrm{SO}_{4} / \mathrm{PEO} / \mathrm{H}_{2} \mathrm{O}$ resultava em um sistema trifásico, onde, a fase mais densa era formada pela mistura $\mathrm{H}_{2} \mathrm{O} / \mathrm{Na}_{2} \mathrm{SO}_{4}$, a fase intermediária era composta por $\mathrm{PEO} /$ $\mathrm{CH}_{2} \mathrm{Cl}_{2}$, enquanto a fase superior era formada por $\mathrm{PEO} / \mathrm{H}_{2} \mathrm{O}$. A adição de n-hexano, a este sistema trifásico, gerava um sistema tetrafásico em que a nova fase era a menos densa e formada por mistura de $\mathrm{CH}_{2} \mathrm{Cl}_{2} / \mathrm{n}$-hexano ${ }^{26}$.

Este comportamento segregativo ou associativo dos componentes formadores dos SABs é geralmente representado em diagramas de fases retangulares ou triangulares. A Figura 1 exemplifica as duas possibilidades.
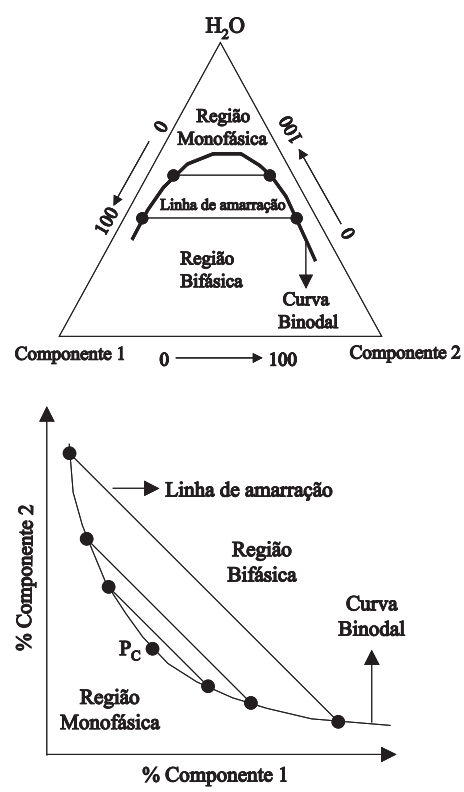

Figura 1. Diagrama de fase para sistemas aquosos bifásicos, expresso em coordenadas triangulares e retangulares

Nestes diagramas encontramos inúmeras informações, todas relacionadas à minimização da energia livre do sistema. Por ex., podemos obter, em quais composições globais o sistema é monofásico ou bifásico, sendo estas duas regiões demarcadas por uma linha denominada curva binodal. Também são representadas as linhas de amarração ("tie lines"), que são retas que ligam pontos no diagrama que representam a composição das duas fases em equilíbrio. Qualquer conjunto de pontos que pertençam à região bifásica e que estejam sobre a 
mesma linha de amarração fornecerá fases superiores que possuirão propriedades termodinâmicas intensivas iguais (densidade, volume molar, entalpia molar, etc.), entretanto, sendo distintas as suas variáveis termodinâmicas extensivas (massa, volume, etc). Aplica-se o mesmo raciocínio para as fases inferiores formadas a partir de composições globais localizadas sobre uma mesma linha de amarração.

O comprimento da linha de amarração, CLA, é um importante parâmetro termodinâmico, geralmente utilizado como variável determinante dos processos de partição dos solutos em SABs formados por diferentes componentes e é calculado pela Equação 3

$\mathrm{CLA}=\left\{\left[\mathrm{C}(\mathrm{POL})_{\mathrm{FS}}-\mathrm{C}(\mathrm{POL})_{\mathrm{FI}}\right]^{2}+\left[\mathrm{C}(\mathrm{SAL})_{\mathrm{FS}}-\mathrm{C}(\mathrm{SAL})_{\mathrm{FI}}\right]^{2}\right\}^{0,5}$

onde $\mathrm{C}(\mathrm{POL})_{\mathrm{FS}}, \mathrm{C}(\mathrm{POL})_{\mathrm{FI}}, \mathrm{C}(\mathrm{SAL})_{\mathrm{FS}}$ e $\mathrm{C}(\mathrm{SAL})_{\mathrm{FI}}$ são as concentrações do polímero e do sal nas fases superior (FS) e inferior (FI), respectivamente. Com o aumento do valor do parâmetro CLA, aumenta-se a diferença entre a fase superior e a inferior, em termos de propriedades termodinâmicas intensivas.

Com base na discussão acima fica claro que o problema básico na análise teórica dos sistemas aquosos bifásicos, em termos da termodinâmica no equilíbrio, é a obtenção de uma expressão analítica para variação da energia livre associada com a formação de uma solução contendo os diferentes polímeros e/ou sais, a partir de seus componentes puros ${ }^{27}$. Esta propriedade termodinâmica é geralmente denominada energia livre de mistura, $\Delta_{\text {mix }} \mathrm{G}^{28}$.

Se as interações que os componentes realizam em solução forem exatamente as mesmas que eles realizam quando puros, mesmo as$\operatorname{sim} \Delta_{\text {mix }}$ G não será igual a zero, pois teremos a contribuição entrópica. Assim, a energia livre de mistura será função das interações resultantes presentes em solução, bem como das diferentes distribuições das moléculas na solução. Estas interações serão expressas para cada componente em termos dos respectivos potenciais químicos. Matematicamente teríamos:

$\Delta_{\text {mix }} G=G_{\text {sol }}-\sum_{i=1}^{3} G_{i}^{*}=\sum_{i=1}^{3} n_{i} \mu_{i}-\sum_{i=1}^{3} n_{i} \mu_{i}^{*}=\sum_{i=1}^{3} n_{i}\left(\mu_{i}-\mu_{i}^{*}\right)$

onde $\mathrm{G}_{\mathrm{sol}}$ é a energia livre da solução, $G_{i}^{*}$ é a energia livre de cada componente puro, $n_{i}$ é a quantidade de substância e $\mu_{i}$ e $\mu^{*}{ }_{i}$ são os potenciais químicos de cada componente em solução e puro, respectivamente.

Termodinamicamente, todas as vezes que $\Delta_{\text {mix }} \mathrm{G}<0$, a mistura dos três componentes produzirá um sistema homogêneo e, caso contrário, o sistema procurará uma nova configuração (a produção de duas fases em equilíbrio), que terá uma menor energia livre. Com temperatura e pressão constantes, a variação da energia livre de mistura ocorrerá apenas se houver variação do potencial químico de um dos componentes quando da adição de qualquer outro componente. Verificamos isto derivando a Equação 4 em relação a cada componente:

$\frac{\partial\left(\Delta_{m i x} G\right)}{\partial n_{i}}=\left(\mu_{i}-\mu_{i}^{*}\right)$

A energia livre de mistura ficará positiva, devido à adição de um dos componentes formadores dos SABs, apenas se neste estado termodinâmico, o potencial químico de um ou mais componentes da solução for maior que o potencial químico do componente quando puro, assim a derivada apresentada na Equação 5 torna-se positiva.

Utilizando a equação clássica, $\Delta_{\text {mix }} \mathrm{G}=\Delta_{\text {mix }} \mathrm{H}-\mathrm{T} \Delta_{\text {mix }} \mathrm{S}$, podemos observar que no termo $\Delta_{\text {mix }} \mathrm{G}$ existem contribuições de natureza entálpica, $\Delta_{\text {mix }} \mathrm{H}$ e outras relacionadas à variação da entropia do sistema, $\Delta_{\text {mix }} \mathrm{S}$. Naturalmente esta divisão é uma aproximação, visto que existe uma conectividade entre a variação da entalpia e da entropia de um sistema. Existem relações similares à Equação 5 para os termos entálpico e entrópico.

A conexão entre estes parâmetros termodinâmicos macroscópicos $\left(\Delta_{\text {mix }} \mathrm{He} \Delta_{\text {mix }} \mathrm{S}\right)$ e as propriedades moleculares é obtida a partir de um tratamento estatístico, utilizando sempre um tipo específico de modelo, entre os quais podemos destacar os de Flory e Huggins ${ }^{29,30}$, de expansão do virial ${ }^{31}$, UNIQUAC ${ }^{32,33}$, de contribuição de grupo $^{34} \mathrm{e} o$ modelo de exclusão volumétrica ${ }^{35}$. Uma excelente revisão que analisa a aplicação destes modelos especificamente aos SABs foi escrita por Cabezas Jr, em $1996^{36}$.

Entretanto, muitas vezes, os novos modelos de termodinâmica estatística quando aplicados especificamente à separação de fases de sistemas contendo polímeros são, na verdade, modificações da teoria original de Flory e Huggins ${ }^{37}$. Em geral, eles foram formulados na tentativa de descrever em maiores detalhes os processos dominantes em soluções poliméricas. No presente artigo faremos uso do modelo de Flory e Huggins por ser mais simples e fornecer aspectos básicos e semiquantitativos dos fenômenos moleculares que ocorrem em sistemas aquosos bifásicos.

$\mathrm{Na}$ aplicação do modelo de Flory e Huggins a sistemas aquosos bifásicos, a solução ternária é representada por uma rede tridimensional de sítios, todos de mesmo volume, ocupados por moléculas do solvente ou segmentos dos polímeros (ou íons). O número de segmentos de cada macromolécula, $\mathrm{X}_{\mathrm{i}}$, é encontrado dividindo-se o volume molar do polímero pelo volume molar do solvente.

Neste modelo de rede, cada sítio tem z faces de contato com sítios adjacentes. Desta forma, a entalpia de mistura é resultante da variação da entalpia associada ao processo de formação de contatos entre os segmentos dos diferentes polímeros e destes segmentos com as moléculas do solvente. Entretanto, é necessário enfatizar que a mistura descrita anteriormente ocorrerá sempre à custa da quebra do contato entre unidades dos componentes de mesma espécie presentes no estado puro. Esta variação de energia por contato, $\Delta \varepsilon$, é dada por:

$\Delta \varepsilon=z_{12}\left[w_{12}-\frac{1}{2}\left(w_{11}+w_{22}\right)\right]+z_{13}\left[w_{13}-\frac{1}{2}\left(w_{11}+w_{33}\right)\right]+z_{23}\left[w_{23}-\frac{1}{2}\left(w_{22}+w_{33}\right)\right](6)$

onde $\mathrm{w}_{\mathrm{ij}}$ é a energia potencial de par devido a interações entre os componentes i e j, e $z_{\mathrm{ij}}$ é o número de coordenação que os componentes $\mathrm{j}$ fazem ao redor de um componente i. Rearranjando os termos da Equação 6 temos:

$\Delta \varepsilon=\Delta \varepsilon_{12}+\Delta \varepsilon_{13}+\Delta \varepsilon_{23}$

em que $\Delta \varepsilon_{\mathrm{ij}}$ é denominada de variação de energia efetiva do par potencial e é igual à metade da energia requerida para formar $2 \mathrm{z}$ pares $i-j$. Estes pares $\mathrm{i}-\mathrm{j}$ são formados a partir do processo de transferência de uma molécula do tipo "i" presente numa fase formada apenas pelo componente i, mais em uma molécula do tipo “j” presente em um sistema contendo apenas $\mathrm{j}$, onde ambas as moléculas estão interagindo com $\mathrm{z}$ vizinhos.

Estes parâmetros de interação poderão ser expressos na forma $\Delta \varepsilon_{\mathrm{ij}}$ ou normalizados em relação à energia térmica, $\chi_{i j}=\frac{\Delta \varepsilon_{i j}}{k T} \cdot \chi_{\mathrm{ij}}$ é denominado parâmetro de Flory-Huggins e leva em consideração a dinâmica de formação e quebra dos contatos devido, principalmente, ao movimento aleatório das moléculas em solução.

A interação será considerada desfavorável entalpicamente caso $\chi_{\mathrm{ij}}$ ou $\Delta \varepsilon_{\mathrm{ij}}$ possuam valores positivos e, favorável caso a interação resultante seja exotérmica (valores negativos). O grande desafio do ponto de vista teórico é estabelecer uma relação entre o número dos diferentes tipos de contato com propriedades macroscópicas que expressem a composição do sistema, tais como fração volumétrica ou 
fração mássica. Encontrando esta relação basta multiplicá-la por $\Delta \varepsilon$, e obteremos o valor de $\Delta_{\text {mix }} \mathrm{H}$.

$\Delta_{\text {mix }} \mathrm{H}=. \Delta \varepsilon, \Gamma\left[\mathrm{n}^{\circ}\right.$ de contatos $]$

onde $\Gamma$ ( $\mathrm{n}^{\circ}$ de contatos) é uma função que estabelece relação entre o número de diferentes contatos e propriedades macroscópicas do sistema. Para um sistema ternário é razoável supormos que a probabilidade de cada tipo de contato é proporcional à quantidade dos componentes envolvidos e de suas frações volumétricas. Assim podemos escrever a Equação 8 como:

$\Delta_{m i x} H=N \sum_{i=1}^{23} \sum_{j=i+1} \Phi_{i} \Phi_{j} \Delta \varepsilon_{i j}$

em que $\Phi_{\mathrm{i}}$ é a fração volumétrica do componente i $\left(\Phi_{i}=\frac{X_{i} n_{i}}{\sum^{3} X_{i} n_{i}}\right)$ na solução resultante, $\mathrm{N}$ é o número total de sítios $\left(N=\sum_{i=1}^{3} X_{i=1} n_{i}\right)$.

Para cálculo da entropia de mistura, leva-se em consideração apenas o número de diferentes maneiras de distribuir as moléculas e os segmentos nos distintos sítios formadores da rede. Esta entropia é denominada entropia configuracional. Este modelo não considera as outras variações entrópicas (conformacionais, rotacionais, eletrônica, etc.). Assim, a contribuição entrópica do processo de mistura é dada pela Equação 10

$\Delta_{m i x} S=-N R \sum_{i=1}^{3} \frac{\Phi_{i}}{X_{i}} \ln \Phi_{i}$

onde $\mathrm{R}$ é a constante dos gases.

$\mathrm{Na}$ Equação 10 só são possíveis valores positivos para $\Delta_{\text {mix }} \mathrm{S}$, indicando assim que, na presença de forças intermoleculares muito fracas, os componentes formadores dos SABs irão sempre se misturar para formar uma única fase. Entretanto, esta contribuição da entropia de mistura para a minimização da energia livre de Gibbs diminui com o aumento do grau de polimerização $\mathrm{X}_{\mathrm{i}}$ do componente i. Assim, os valores de $\Delta_{\text {mix }} \mathrm{S}$ envolvendo sistemas contendo macromoléculas serão sempre menores que os valores da variação de entropia associada à mistura de moléculas pequenas, da mesma forma que uma macromolécula de massa molar $100.000 \mathrm{~g} \mathrm{~mol}^{-1}$ promoverá um ganho entrópico menor que um polímero, quimicamente semelhante ao anterior, mas de massa molar igual $1000 \mathrm{~g} \mathrm{~mol}^{-1}$. É por isto que a mistura etanol e glicose, moléculas parecidas com as unidades monoméricas do poli(óxido de etileno) e da dextrana, será entropicamente mais favorável que a mistura das respectivas macromoléculas. Sistemas formados pela mistura de $20 \%(\mathrm{~m} / \mathrm{m}) \mathrm{de}$ etanol e $20 \%(\mathrm{~m} / \mathrm{m})$ de glicose a $25{ }^{\circ} \mathrm{C}$ são completamente miscíveis, enquanto que sistemas formados na mesma temperatura pela mistura de $0,5 \%$ de dextrana e 5,0\% de PEO formam sistemas aquosos bifásicos. Em ambos os sistemas, a interação entálpica resultante é desfavorável, mas apenas no sistema macromolecular é que esta pequena energia repulsiva consegue superar a variação de entropia favorável, levando à formação de um sistema bifásico.

A combinação dos dois termos discutidos anteriormente, a entalpia e a entropia de mistura, na clássica equação da termodinâmica, $\Delta_{\text {mix }} \mathrm{G}=\Delta_{\text {mix }} \mathrm{H}-\mathrm{T} \Delta_{\text {mix }} \mathrm{S}$, fornece a Equação 11

$\Delta_{m i x} G=N \sum_{i=1 j=i+1}^{2} \Phi_{i}^{3} \Phi_{j} \Delta \varepsilon_{i j}-N R T \underset{i=1}{\stackrel{3}{\sum} \frac{\Phi_{i}}{X_{i}} \ln \Phi_{i}}$

Caso tenhamos valores de $\Delta \varepsilon_{\mathrm{ij}}$ ou $\chi_{\mathrm{ij}}$ medidos ou calculados para um dado sistema em função da temperatura e concentração, pode- mos aplicar estes valores na Equação 11 e minimizando-a obteremos os dados referentes ao diagrama de fase, incluindo as composições das fases em equilíbrio, a curva binodal, o ponto crítico, etc. Infelizmente estes dados são escassos, e muitas vezes a minimização da função $\Delta_{\text {mix }}$ G não é tarefa simples, levando à necessidade de aplicar novos modelos ${ }^{38-42}$.

\section{PARTIÇÃO DE PROTEÍNAS EM SABS}

Em geral, a distribuição de proteínas entre as duas fases aquosas dos SABs é caracterizada por um parâmetro denominado coeficiente de partição, $\mathrm{K}_{\mathrm{p}}$, definido como $^{43-47}$

$K_{P}=\frac{a_{P, S}}{a_{P, I}} \cong \frac{c_{P, S}}{c_{P, I}}$

onde $\mathrm{a}_{\mathrm{p}, \mathrm{i}}$ e $\mathrm{c}_{\mathrm{p}, \mathrm{i}}$ são, respectivamente, a atividade e a concentração da proteína nas diferentes fases ( $\mathrm{S}=$ superior ou $\mathrm{I}=$ inferior). A Equação 12 enfatiza o ponto de vista termodinâmico, em que os parâmetros "concentração" presentes nesta equação, deveriam ser substituídos pela "atividade" da proteína nas duas fases. É importante destacar que muitas vezes, as medidas experimentais do coeficiente de partição das proteínas têm revelado uma dependência de $\mathrm{K}_{\mathrm{p}}$ em relação à concentração da mesma indicando tratar-se de um coeficiente aparente, não constituindo, desta forma, um parâmetro que caracterize um sistema em equilíbrio termodinâmico. Além disto, a dependência de $\mathrm{K}_{\mathrm{p}}$ em relação à concentração pode também indicar que está ocorrendo a partição de diferentes espécies químicas formadas, por ex., pela agregação das proteínas. Entretanto, para experimentos em que a concentração da proteína é muito baixa (regime de diluição infinita), os valores da concentração e da atividade tornam-se muito próximos ${ }^{48}$.

Esta distribuição desigual da biopartícula entre as duas fases é resultante de um intrincado e delicado balanço de interações entre a proteína e as outras espécies (polímeros, $\mathrm{H}_{2} \mathrm{O}$ e/ou sais inorgânicos) presentes nas duas fases que coexistem em equilíbrio ${ }^{49}$.

Inúmeras propriedades físico-químicas do sistema e do biopolímero determinam o valor de $\mathrm{K}_{\mathrm{p}}$. Exemplos de fatores associados à proteína que influenciam sua distribuição entre as fases são $0^{50-55} \mathrm{o}$ tamanho, a conformação (estruturas secundária, terciária e quaternária) e a composição (estrutura primária), presença de carga elétrica e hidrofobicidade. Além disto, propriedades importantes das fases também contribuem nesta distribuição, como por ex., a natureza química dos componentes formadores dos $\mathrm{SABs}^{56,57}$, a massa molar e a concentração dos polímeros ${ }^{58,59}$, a presença de ligantes ao longo da cadeia polimérica que possam interagir especificamente com sítios da proteína ${ }^{60,61}, \mathrm{pH}$ e temperatura ${ }^{62,63}$. Além do mais, a adição de diferentes sais inorgânicos a estes sistemas bifásicos produz uma diferença de potencial elétrico na interface, que influencia marcantemente a partição de proteínas carregadas eletricamente ${ }^{64-67}$. Este potencial eletrostático ocorre devido à distribuição desigual dos cátions e dos ânions entre as fases enriquecidas pelos diferentes polímeros $^{68}$.

É possível relacionar o parâmetro obtido experimentalmente, $\mathrm{K}_{\mathrm{p}}$, com a variação da energia livre de Gibbs associada ao processo de transferência da proteína da fase inferior para a fase superior, $\Delta_{\mathrm{t}} \mathrm{G}=-$ $\mathrm{RT} \operatorname{lnK}$.

Esta função termodinâmica de transferência, $\Delta_{t} \mathrm{G}$, está relacionada com a interação da proteína com os diferentes polímeros e/ou sais presentes nas fases e também com as interações que cada componente formador dos SABs realiza entre eles ${ }^{69}$. As medidas do coeficiente de partição devem ser realizadas em regime de diluição infinita onde se procura evitar a contribuição da interação proteína-pro- 
teína para o $\Delta_{\mathrm{t}} \mathrm{G}$. Entretanto, é bem conhecido que mesmo em situações de baixíssima concentração as proteínas podem se associar sob certas condições, particularmente perto do seu ponto isoelétrico e/ou na presença de outros polímeros ${ }^{70}$.

Assim, a contribuição ou não da interação proteína-proteína para o coeficiente de partição precisa ser verificada, principalmente para aquelas proteínas que são caracteristicamente auto-associativas. Outra motivação importante para a determinação do coeficiente de partição, de uma proteína em baixa concentração, é que nestas condições a presença da proteína não afeta o estado termodinâmico do SABs aplicado para a purificação. Uma concentração típica que tem confirmado a assertiva anterior é $1 \mathrm{mg} / \mathrm{mL}^{71}$.

A estratégia termodinâmica para cálculo de $\mathrm{K}_{\mathrm{P}}$ consiste em se obter o potencial químico da proteína em cada fase do SABs. Em geral, este parâmetro é fornecido pelas relações termodinâmicas apresentadas abaixo

$\mu_{P}^{S}=\left(\frac{\partial \Delta_{\text {mix }} G}{\partial n_{P}^{S}}\right)_{T, P, n_{j} \neq n_{i}}$

$\mu_{P}^{S}=\mu_{P}^{0}+R T \ln \Phi_{P}^{S}+\left(\mu_{P}^{e x}\right)^{S}$

Nas Equações 13 e 14, $\mu_{P}^{S}$ é o potencial químico da proteína na fase superior, enquanto $\mu^{0}{ }_{p}$ é potencial químico da proteína pura que se encontra na mesma temperatura do SABs e $\left(\mu^{\mathrm{ex}}\right)^{\mathrm{S}}$ é o potencial químico de excesso da proteína na fase superior.

Para a aplicação das duas equações anteriores, sobre os dados de equilíbrio dos sistemas aquosos bifásicos, visando a obtenção do coeficiente de partição, é necessário supormos que a adição da proteína não altera o estado termodinâmico inicial (antes da adição) do sistema. A aplicação destas relações termodinâmicas (Equações $13 \mathrm{e}$ 14), na Equação 11 nos fornece a seguinte vinculação entre o potencial químico de excesso e a constante de partição ${ }^{72}$

$\ln K_{P}=\frac{1}{R T}\left[\left(\mu_{P}^{e x}\right)^{S}-\left(\mu_{P}^{e x}\right)^{i}\right]$

Na Equação 15 o potencial químico de excesso expressa a entropia de mistura não ideal de todos os componentes formadores dos SABs, bem como todas as interações entálpicas entre todos os componentes no sistema, inclusive as da proteína com os todos os compostos que formem cada fase.

A combinação das Equações 13 e 15, e a posterior substituição do resultado na Equação 11 leva a uma relação matemática que nos fornece a contribuição entrópica para o processo de partição da proteína $^{72}$. Nesta dedução matemática considera-se inexistente a contribuição entálpica para a variação da energia livre de Gibbs de mistura. A Equação final é apresentada abaixo

$\ln K_{P}=\frac{M_{P}}{\rho}\left(\frac{n^{S}}{V^{S}}-\frac{n^{i}}{V^{i}}\right)$

onde, $M_{p}$ é grau de polimerização da proteína, $\rho$ é o número de sítios por unidade de volume de todo o sistema bifásico, $\mathrm{n}^{\mathrm{s}}$ é o número total de moléculas na fase superior, enquanto que $\mathrm{n}^{\mathrm{i}}$ é o número total de moléculas presentes na fase inferior e $\mathrm{V}^{\mathrm{s}}$ e $\mathrm{V}^{\mathrm{i}}$, os volumes das fases superior e inferior, respectivamente.

A Equação 13 nos mostra que a contribuição entrópica para a partição da proteína só aparecerá caso exista uma diferença em termos de densidade numérica entre as duas fases. Como força motriz, a entropia levará a proteína a particionar para a fase com maior número de moléculas por unidade de volume, pois nesta fase haverá um maior número de formas de distribuir as moléculas espacialmen- te, aumentando assim a contribuição da entropia configuracional para a minimização do parâmetro termodinâmico $\Delta_{\text {mix }}$ G. Na maioria dos SABs atualmente conhecidos, são as moléculas de água que estão presentes em grande maioria numérica, em ambas as fases e, por isto, é este o componente determinante para a densidade numérica das diferentes fases. Assim, em linhas gerais, a contribuição entrópica fará a proteína tender a particionar para a fase com maior conteúdo de moléculas de água por unidade de volume.

Durante a partição de proteínas em sistemas aquosos bifásicos formados pela mistura de eletrólito/polímero, forças motrizes de natureza entrópica atuarão no processo forçando o biopolímero a se concentrar na fase rica em sal, visto ser esta a que contém maior densidade numérica ${ }^{73-75}$. A concentração de proteínas na fase rica em polímero só ocorrerá nestes SABs (sal/polímero) caso existam interações entálpicas contribuindo para este comportamento de partição como, por ex., quando há interações específicas entre proteína e polímero ${ }^{76}$.

Para sistemas com densidade numérica das fases muito próximas, isto é, sistemas onde o fator entrópico pouco contribui, é a entalpia que determinará o comportamento de partição da proteína. Neste caso, o valor de $\mathrm{K}_{\mathrm{p}}$ depende fundamentalmente da diferença entre os valores que a entalpia molar parcial da proteína, $\bar{h}_{P}$, apresentará nas duas distintas fases. No estado termodinâmico em que não ocorra interação proteína-proteína, a entalpia molar parcial será dada pela equação ${ }^{72}$

$\bar{h}_{P}=\left(\frac{\partial \Delta_{\text {mix }} H}{\partial n_{P}}\right){ }_{T, P, n_{j} \neq n_{i}}$

A aplicação da Equação 17 na Equação 14 e, considerando que na ausência de contribuição entrópica, $\mu^{\mathrm{ex}}=\bar{h}_{P}$, obteremos após alguns rearranjos a seguinte relação entre $K_{p}$ e a composição das fa$\operatorname{ses}^{72}$

$\ln K_{P}=-\frac{M_{P}}{R T}\left[\sum_{i=1(i \neq p)}^{3}\left(\Phi_{i}^{S}-\Phi_{i}^{I}\right) \varepsilon_{i p}-\sum_{i=1(i \neq p)}^{2} \sum_{j=i+1(j \neq p)}^{3}\left(\Phi_{i}^{S} \Phi_{j}^{S}-\Phi_{i}^{i} \Phi_{j}^{i}\right) \varepsilon_{i j}\right.$

A Equação 18 expressa todas as interações entálpicas que ocorrem dentro do sistema aquoso bifásico. Podemos separar a contribuição entálpica para a partição da proteína em dois termos. O primeiro somatório é a contribuição da interação da proteína com cada componente formador das fases, em que $\varepsilon_{\mathrm{ip}}$ representa a energia resultante da formação de um par entre proteína e o componente i. Neste termo entálpico, a energia resultante é proporcional à diferença de composição entre as duas fases. Considerando apenas a interação da proteína com os diferentes componentes, presentes no $\mathrm{SABs}$, a proteína particionará para a fase que contem maior concentração do componente com o qual ela interaja mais entalpicamente, isto é, tenha o valor de $\varepsilon_{\mathrm{iP}}$ mais negativo ( ou menos positivo).

O segundo termo na Equação 18 representa a variação da energia associada à transferência da proteína da fase inferior para a superior, sendo a mesma, entretanto, independente da interação da proteína com os componentes de cada fase. Este termo energético só depende da interação que ocorre entre os componentes formadores das fases, que pode ser interpretado como um conteúdo energético de cada fase. Esta variação de energia está presente porque ao se transferir da fase inferior, a proteína libera um volume que permite a interação entre os componentes presentes na fase, enquanto que seu deslocamento para a fase superior possibilitará a formação de uma cavidade, impossibilitando a interação entre os componentes da fase superior.

Desta forma, a contribuição deste termo entálpico para o comportamento de partição da biopartícula faz com que a proteína se difunda para a fase onde é menor o gasto energético para formação da cavidade em que a mesma será alojada. 
Este modelo teórico, as Equações 15-18, nos permite interpretar qualitativamente algumas influências que certas características dos SABs têm sobre o comportamento de partição das proteínas. É bem conhecido que concentração, massa molar e tipo de polímero formador das fases influenciam decisivamente o comportamento de extração das biopartículas.

Em relação à influência que a composição das fases exerce sobre a partição das proteínas, algumas tendências podem ser destacadas. Quanto menor o comprimento da linha de amarração, CLA, associada a uma dada composição global do sistema, mais próximo a 1 será o valor do coeficiente de partição, portanto, menos seletiva a extração. Segundo o modelo, nesta condição termodinâmica (pequenos valores de CLA), a distribuição igualitária da proteína entre as duas macro-regiões ocorre porque com a mínima diferença de concentração dos constituintes entre as fases será ínfima também a diferença entre elas em termos de densidade numérica, auto-energia e energia de interação. Entretanto, com o aumento do CLA, a transferência da proteína tende a uma das fases, isto é, o valor de $\mathrm{K}_{\mathrm{p}}$ torna-se cada vez mais distante de 1, para mais ou para menos. Este comportamento é resultante do aumento na diferença da composição entre as fases que gera, conseqüentemente, o incremento das contribuições entrópicas (Equação 13) e entálpicas (Equação 15) na transferência da proteína para uma das fases.

Em geral, o aumento da massa molar de um polímero que é rico em uma fase acaba diminuindo a tendência de partição da proteína para a mesma fase. Naturalmente, este aumento no grau de polimerização do polímero formador do SABs acaba diminuindo a entropia configuracional da fase enriquecida neste polímero, reduzindo o valor da entropia de transferência da proteína para esta fase.

Outra propriedade físico-química que influencia marcantemente a partição de uma proteína é o $\mathrm{pH}$. A maioria dos trabalhos que investigaram esta correlação foram realizados em sistemas aquosos bifásicos com composição global fixa, às vezes, com adição de eletrólitos ${ }^{19}$. A concentração hidrogeniônica afeta a transferência das proteínas, porque estes biopolímeros contêm uma grande variedade de grupos ácidos e básicos com diferentes valores de $\mathrm{pK}_{\mathrm{a}}$, resultando em cargas elétricas que são funções dos valores de $\mathrm{pH}$. Esta densidade de carga elétrica na superfície da proteína pode modificar sua conformação, bem como processos de associação ou dissociação entre as macromoléculas presentes no sistema. Todos estes processos que ocorrem com a proteína modificam sua interação com os componentes do sistema bifásico, alterando seu comportamento de partição. $\mathrm{O}$ $\mathrm{pH}$, indiretamente, pode afetar a partição de uma proteína e modificar a composição das fases dos SABs, seja a posição da linha binodal, seja o comprimento da linha de amarração.

Os primeiros trabalhos que investigaram o efeito do tamanho do soluto sobre seu comportamento de partição indicavam que moléculas pequenas, como aminoácidos e glicosídios, tendiam a distribuirse igualmente $(K \cong 1)$ nos sistemas aquosos bifásicos enquanto macromoléculas, principalmente grandes proteínas e ácidos nucléicos, tendiam a se concentrar em uma das fases ${ }^{77}$. Trabalhos posteriores possibilitaram mostrar que não era uma tendência geral. Albertsson e colaboradores ${ }^{78}$ mostraram que a enzima $\beta$-galactosidase, com massa molar igual a $540000 \mathrm{~g} \mathrm{~mol}^{-1}$, particiona mais igualmente $(\mathrm{K}=0,38)$ que a pequena proteína citocromo $\mathrm{C}$, de massa molar $12384 \mathrm{~g} \mathrm{~mol}^{-1}(\mathrm{~K}=0,18)$ no SABs Dex70-PEG-8000 contendo o tampão $0,01 \mathrm{~mol} \mathrm{~L}^{-1}$ de fosfato de sódio, $\mathrm{pH}$ 6,8. Após sucessivos trabalhos utilizando diferentes proteínas, a comunidade científica reconheceu a dificuldade em separar os efeitos devidos ao tamanho da partícula daqueles relacionados à estrutura química.

Com o objetivo de superar esta dificuldade, Zaslavsky e colaboradores $^{79,80}$ estudaram a partição de séries homologas de polímeros sintéticos. Foram investigados o comportamento de partição de dife- rentes massas molares de polietilenoglicol(PEG), poliacrilami$\mathrm{da}(\mathrm{PAAm})$ e polivinilpirrolidona(PVP) em SABs formados por Dex 70-Ficoll 400 contendo $0,15 \mathrm{~mol} \mathrm{~kg}^{-1}$ de $\mathrm{NaCl} \mathrm{e} 0,01 \mathrm{~mol} \mathrm{~kg}^{-1}$ de tampão fosfato, $\mathrm{pH} 7,4$. Mesmo neste estudo, onde os efeitos eram apenas relacionados ao tamanho da partícula, diferentes comportamentos foram observados. O polímero PEG teve o coeficiente de partição aumentado com o aumento da massa molar, enquanto os polímeros PVP e PAAm apresentaram um comportamento inverso.

A interpretação deste efeito da massa molar (tamanho da biopartícula) sobre o comportamento de partição da proteína, segundo o modelo descrito no presente artigo, deve considerar as duas forças motrizes termodinâmicas. Como podemos verificar pela Equação 16, nos casos em que o fator entrópico predomina sobre o fator entálpico, o aumento do tamanho da biopartícula levará sempre a uma distribuição mais desigual da proteína, com variação proporcional à sua massa molar. Entretanto, a ocorrência de um comportamento inverso deve-se à contribuição das interações entálpicas que a proteína realiza com os diferentes compostos que forman as fases (Equação 18).

Apesar dos avanços obtidos nos estudos sobre os sistemas aquosos bifásicos, ainda é reduzido o número de casos em que tais sistemas foram aplicados industrialmente ${ }^{81}$. Além disso, uma consulta aos bancos de patente forneceu apenas oito registros, a maioria relacionados à aplicação dos $\mathrm{SABs}$ na purificação de proteínas presentes em misturas complexas produzidas geralmente por culturas de microorganismos. Por ex., Tjerned e Sivars ${ }^{82}$ propuseram um método de extração por afinidade, para a proteína de membrana, $\mathrm{CytBO}_{3}$, em um SABs formado pela mistura de uma solução aquosa de fosfato e uma solução aquosa de PEG-IDA (polietilenoglicol-ácido iminodiacético). Segundo os autores desta patente, este método aumentou a seletividade, o rendimento e o fator de purificação em relação aos métodos tradicionais utilizados para purificação desta proteína.

\section{CONCLUSÃO}

Atualmente é alta a demanda por métodos de extração e purificação de biopartículas (proteínas, enzimas, células, etc.) que sejam eficientes e economicamente viáveis. Nessa linha, os sistemas aquosos bifásicos constituem um potencial. Entretanto, é notório que os SABs apesar da intensa aplicação em escala laboratorial para purificação de enzimas e proteínas, não alcançaram ampla aplicação comercial, tendo talvez como possíveis causas o custo dos polímeros formadores das fases e/ou o comportamento complexo (inúmeras variáveis interferindo de forma cinegética ou antagônica) de partição apresentados nestes sistemas bifásicos. Além disso, para sua aplicação sistemática, é necessária maior compreensão dos fatores que determinam sua formação e a partição de solutos específicos. Contudo, a urgente demanda por parte da indústria biotecnológica, com objetivo de trazer para o mercado consumidor produtos que possuam alto valor agregado, com ampla aplicação médica, certamente motivará a aplicação em escala industrial dos SABs.

Conseqüentemente, processos utilizando SABs em etapas de recuperação primária de produtos de interesse biotecnológico serão uma importante opção para novos bioprocessos associados à indústria farmacêutica.

\section{REFERÊNCIAS}

1. Silva, M. E.; Franco, T. T.; Braz. J. Chem. Eng. 2000, 17, 1.

2. Pessoa, A.; Kolikian, B. V.; Purificação de Produtos Biotecnológicos, Manole: São Paulo, 2005

3. Selber, K.; Tjerneld, F.; Collen, A.; Hyytia, T.; Nakari-Setala, T.; Bailey, M.; Fagerstrom, R.; Kan, J.; van der Laan, J.; Penttila, M.; Kula, M. R.; Process Biochem. 2004, 39, 889.

4. Beijerinck, M. W.; Zbl. Bakt. II Natur. 1896, 627, 698 
5. Beijerinck, M. W.; Kolloid Z. Z. Polym. 1910, 7, 16.

6. Ostwald, W.; Hertel, R. H.; Kolloid Z. Z. Polym. 1929, 47, 258.

7. Ostwald, W.; Hertel, R. H.; Kolloid Z. Z. Polym. 1929, 47, 357.

8. Dobry, A.; Boyer-Kawenoki, F.; J. Polym. Sci. 1947, 2, 90.

9. Dobry, A.; Bull. Soc. Chim. Belg. 1948, 57, 280.

10. Tiselius, A.; Porath, J.; Albertsson, P.A.; Science 1963, 141, 13.

11. Kim, C. W.; Rha, C. Y.; Phys. Chem. Liq. 2000, 38, 25.

12. Mishima, K.; Matsuyama, K.; Ezawa, W.; Taruta, Y.; Takarabe, S.; Nagatini, M.; J. Chromatogr., B: Anal. Technol. Biomed. Life Sci. 1998, 711, 313.

13. Kaul, A.; Pereira, R. A. M.; Asenjo, J. A.; Merchuk, J. C.; Biotechnol. Bioeng. 1995, 48, 246

14. Venancio, A.; Almeida, C.; Teixeira, J. A.; J. Chromatogr., B: Anal. Technol. Biomed. Life Sci. 1996, 680, 131.

15. Save, S. V.; Pangarkar, V. G.; Kumar, S. V.; Biotechnol. Bioeng. 1993, 41, 72

16. Ryden, J.; Albertsson, P. A.; J. Colloid Interface Sci. 1971, 37, 219.

17. Bamberg, S.; Geoffrey, V.; Seaman, F.; Sharp, K. A.; Brooks, D. E.; J. Colloid Interface Sci. 1984, 99, 194.

18. Brooks, D. E.; Walter, H.; Fisher, D.; Partitioning in Aqueous Two Phase Systems: Theory, Methods, Uses, and Applications to Biotechnology, Academic Press: Orlando FL, 1985.

19. Zaslavsky, B. Y.; Aqueous Two Phase Partitioning, Physical Chemistry and Bioanalytical Applications, Marcel Dekker: New York, 1995.

20. Zhang, K. W.; Karlstrom, G.; Lindman, B.; J. Phys. Chem. 1994, 98, 4411

21. da Silva, L. H. M.; Loh, W.; J. Phys. Chem. B 2000, 104, 10069.

22. Guan, Y.; Treffry, T. E.; Lilley, T. H.; J. Chromatogr., A 1994, 668, 31.

23. Jiang, J. W.; Prausnitz, J. M.; J. Phys. Chem. B 2000, 104, 7197.

24. Thalberg, K.; Lindman, B.; Colloids Surf. 1993, 76, 283.

25. Sakurai, K.; Shinkai, S.; Carbohydr. Res. 2000, 324, 136.

26. da Silva, L. H. M.; Loh, W.; Chem. Commun. 1998, 7, 78

27. Prausnitz, J. M.; Tavares, F. W.; AICHE J. 2004, 50, 2635.

28. Rowlinson, J. S.; Swinton, F. L.; Liquids and liquids mixtures, Butterworth: London, 1982

29. Flory, P. J.; Principles of Polymer Chemistry, Cornell University Press: Ithaca, 1953

30. Pessoa, P. A.; Mohamed, R. S.; Process Biochem. 2004, 39, 2075.

31. Haraguchi, L. H.; Mohamed, R. S.; Loh, W.; Pessoa, P. A.; Fluid Phase Equilib. 2004, 215, 1.

32. Hartounian, H.; Sandler, S. I.; Kaler, E. W.; Ind. Eng. Chem. Res. 1994, $33,2288$.

33. da Silva, L. H. M.; Meirelles, A. J. A.; Carbohydr. Polym. 2000, 42, 273.

34. Haghtalab, A.; Mokhtarani, B.; J. Chem. Thermodyn. 2005, 37, 289

35. Guan, Y.; Lilley, T. H.; Garcialisbona, M. N.; Treffry, T. E.; Pure Appl. Chem. 1995, 67, 955 .

36. Jr Cabezas, H.; J. Chromatogr., B: Anal. Technol. Biomed. Life Sci. 1996 680, 3.

37. Pessoa, P. A.; Mohamed, R. S.; Can. J. Chem. Eng. 2004, 82, 530.

38. Xu, X.; Madeira, P. P.; Macedo, E. A.; Chem. Eng. Sci. 2004, 59, 1153.

39. Kan, P.; Lee, C. J.; Ind. Eng. Chem. Res. 1996, 35, 2015.

40. Baughman, D. R.; Liu, Y. A.; Ind. Eng. Chem. Res. 1994, 33, 2668.

41. Haghtalab, A.; Mokhtarani, B.; Maurer, G.; J. Chem. Eng. Data 2003, 48, 1170 .

42. Wu, Y. T.; Zhu, Z. Q.; Lin, D. Q.; Li, M. A.; Fluid Phase Equilib. 1999, 154,109

43. Waziri, S. M.; Abu-Sharkh, B. F.; Ali, S. A.; Biotechnol. Prog. 2004, 20 526.

44. Jiang, J. W.; Prausnitz, J. M.; J. Phys. Chem. B 2000, 104, 7197.

45. Xiao, J. X.; Huang, J. B.; He, X.; Bao, Y. X.; Zhu, B. Y.; Acta Chim. Sinica 2000, 58, 922 .

46. Xiao, J. X.; Sivars, U.; Tjerneld, F.; J. Chromatogr., B: Anal. Technol. Biomed. Life Sci. 2000, 743, 327.

47. Bim, M. A.; Franco, T. T.; J. Chromatogr., B: Anal. Technol. Biomed. Life Sci. 2000, 743, 349.
48. Prausnitz, J. M.; Lichtenthaler, R. N.; Azevedo, E. G.; Molecular Thermodynamics of Fluid-Phase Equilibria, Prentice-Hall: Englewood Cliffs, 1986

49. Farruggia, B.; Nerli, B.; Pico, G.; J. Chromatogr., B: Anal. Technol. Biomed. Life Sci. 2003, 798, 25.

50. Collen, A.; Ward, M.; Tjerneld, F.; Stalbrand, H.; J. Chromatogr., A 2001, 910, 275.

51. Ramsch, C.; Kleinelanghorst, L. B.; Knieps, E. A.; Thommes, J.; Kula, M. R.; Biotechnol. Bioeng. 2000, 69, 83.

52. Berggren, K.; Tjerneld, F.; Veide, A.; Bioseparation 2000, 9, 69.

53. Jonsson, M.; Johansson, H. O.; J. Chromatogr., A 2003, 983, 133.

54. Nilsson, A.; Johansson, H, O.; Mannesse, M.; Egmond, M. R.; Tjerneld, F.; Biochim. Biophys. Acta 2002, 1601, 138

55. Collen, A.; Ward, M.; Tjerneld, F.; Stalbrand, H.; J. Biotechnol. 2001, 87, 179.

56. da Silva, L. H. M.; Meirelles, A. J. A.; Carbohyd. Polym. 2001, 46, 267.

57. Johansson, H. O.; Lundh, G.; Karlstrom, G.; Tjerneld, F.; Biochim. Biophys. Acta 1996, 1290, 289.

58. Huddleston, J.; Abelaira, J. C.; Wang, R. D.; Lyddiatt, A.; J. Chromatogr., B: Anal. Technol. Biomed. Life Sci. 1996, 680, 31.

59. Hartounian, H.; Kaler, E. W.; Sandler, S. I.; Ind. Eng. Chem. Res. 1994, 33, 2294.

60. Sharp K. A.; Yalpani, M.; Howard, S. J.; Brooks, D. E.; Anal. Biochem. 1986, 154,110

61. Brooks, D. E.; Lutwyche, P. ACS Symp. Ser. 1994, 207, 62

62. Waziri, S. M.; Abu-Sharkh, B. F.; Ali, S. A.; Biotechnol. Prog. 2004, 20, 526.

63. de Belval, S.; le Breton, B.; Huddleston, J.; Lyddiatt, A.; J. Chromatogr., B: Anal. Technol. Biomed. Life Sci. 1998, 711, 19.

64. Farruggia, B.; Rigatuso, R.; Capezio, L.; Diez, V.; Pico, G.; J. Chromatogr., B: Anal. Technol. Biomed. Life Sci 2004, 809, 301.

65. Fan, W. Y.; Glatz, C. E.; Sep. Sci. Technol. 1999, 34, 423

66. Bodhankar, S. S.; Gaikar, V. G.; J. Chem. Technol. Biotechnol. 1998, 73, 251.

67. Andrews B. A.; Asenjo J.A.; J. Chromatogr., B: Anal. Technol. Biomed. Life Sci 1996, 685, 15.

68. Pfennig, A.; Schwerin, A.; Gaube, J.; J. Chromatogr., B: Anal. Technol. Biomed. Life Sci 1998, 711, 45.

69. Abbott, N. L.; Blankschtein, D.; Hatton, A. T.; Macromolecules 1991, 24 , 4334.

70. Clark, L. A.; Protein Sci. 2005, 14, 653.

71. Palomares, M. R.; J. Chromatogr., B: Anal. Technol. Biomed. Life Sci 2004, 807,3 .

72. Johansson, H. O.; Karlström, G.; Tjerneld, F.; Haynes, C. A.; J. Chromatogr., B: Anal. Technol. Biomed. Life Sci 1998, 711, 3.

73. da Silva, L. H. M.; Meirelles, A. J. A.; J. Chem. Eng. Data 2001, 46, 251.

74. Sarmento, M. J.; Pires, M. J.; Cabral, J. M. S.; Airesbarros, M. R.; J. Chromatogr., A 1994, 668, 117.

75. Mayerhoff, Z. D. V. L.; Roberto, I. C.; Franco, T. T.; Biochem. Eng. J. 2004, 18,217

76. Jonsson, M.; Johansson, H. O.; J. Chromatogr., A 2003, 983, 133.

77. Albertsson, P. A.; Partition of Cell Particles and Macromolecules, $2^{\text {nd }}$ ed., Wiley- Interscience: New York, 1971.

78. Albertsson, P. A.; Cajarville, A.; Brooks, D. E.; Tjerneld, F.; Biochim. Biophys. Acta 1987, 926, 87.

79. Zaslavsky, B. Y.; Baevskii, A. V.; Rogozhin, S. V.; Gedrovich, A. V.; Shishkov, A. V.; Gasanov, A. A.; Masimov, A. A.; J. Chromatogr., B: Anal. Technol. Biomed. Life Sci 1984, 285, 63

80. Zaslavsky, B. Y.; Miheeva, L. M.; Rogozhin, S. V.; Davidovich, Y. A.; Gedrovich, A. V.; Shishkov, A. V.; Gasanov, A. A.; Masimov, A. A.; J. Chromatogr., B: Anal. Technol. Biomed. Life Sci. 1984, 291, 203

81. Rito-Palomares, M.; J. Microb. Biotechnol. 2002, 12, 535.

82. Tjerned, F.; Sivars, U.; US pat. 2003049819-Al 1999. 